\title{
A Great Imitator: Sarcoidosis
}

\author{
Lena A. Omar ${ }^{\mathrm{a}}$, Emmy Hebert ${ }^{\mathrm{a}}$, Saspin Nakornsri ${ }^{\mathrm{b}}$, \\ Hythem A. Omar, c
}

\begin{abstract}
With the practice of medicine becoming more "cook-book" oriented, it is easy for physicians to develop tunnel vision. It is important for us to be reminded that differentials still exist and that certain multi-organ diseases can easily and often mimick more sinister pathology. This case report presents a patient with a history of cervical cancer who presented with a clinical picture of widespread metastatic disease. Workup eventually lead to a diagnosis of multi-organ sarcoidosis, still a serious condition but less ominous than the alternative. A potential relationship between sarcoid and malignancy is also discussed.
\end{abstract}

Keywords: Sarcoidosis; Malignancy; Noncaseating granulomas; Sclerotic lesions; Adenopathy

\section{Introduction}

We all recall those certain diseases that were labeled "the great mimickers or imitators" by our professors in medical school. Over time, many of us forget our mentors' wise words and need a little reminder. Such was the case when a 54-year-old female with a history of stage II B cervical cancer presented with apparent widespread metastatic disease only to be diagnosed with sarcoid. Sarcoid is a multisystem disease that can truly confound the clinical picture yielding misdiagnoses and mistreatments. A possible relationship be-

\footnotetext{
Manuscript accepted for publication April 25, 2013

${ }^{a}$ UT Southwestern Medical Center, 5323 Harry Hines Boulevard, MC 8896, Dallas, TX 75390, USA

${ }^{\mathrm{b}}$ Dallas County Hospital District, 303 E. Overton Rd. Dallas, TX 75216, USA

${ }^{\mathrm{c}}$ Corresponding author:Hythem A. Omar, UT Southwestern Medical Center, 5323 Harry Hines Boulevard, MC 8896, Dallas, TX 75390, USA. Email: Hythem.omar@utsouthwestern.edu
}

doi: http://dx.doi.org/10.4021/jmc1280w tween sarcoid and malignancy has been postulated, especially with gynecologic oncologies. Because sarcoid can present variably (even within a single organ), the diagnosis can be challenging and even confused with more grave pathology. One can imagine the emotional consequences that may occur with misdiagnoses, especially in our case.

\section{Case Report}

A 54-year-old female with a history of stage II B cervical cancer treated by chemo/radiation therapy presented with gradually worsening cough, shortness of breath, and low back pain one year after treatment. Symptoms had been present for a few months and she had lost approximately 20 pounds over the past year. She was treated for "walking pneumonia" and received multiple courses of antibiotics without improvement. Pertinent negatives included no fever, chills, nausea or vomiting, hemoptysis or sputum production. She also denied vaginal bleeding or discharge. Social and family histories were noncontributory.

The patient was in no acute distress with stable vital

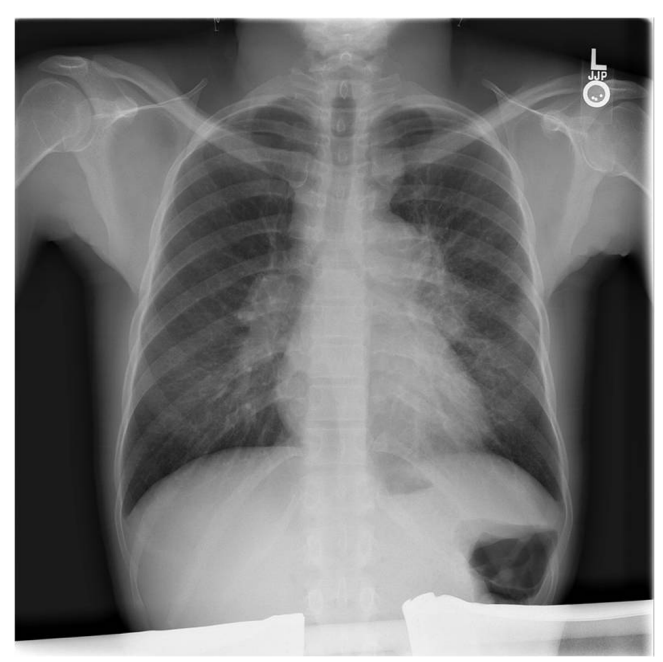

Figure 1. Chest radiograph illustrating mediastinal and bilateral hilar adenopathy. 


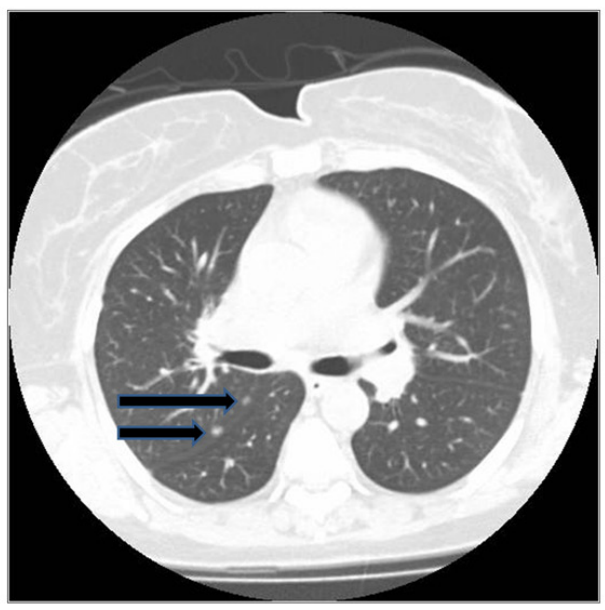

a

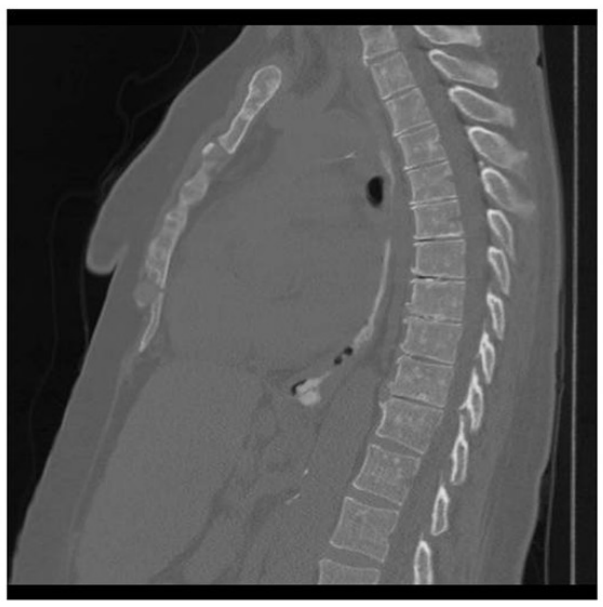

b

Figure 2. Computed tomography of the chest. (a). Axial image through the chest in lung windows illustrating pulmonary nodules (arrows) abutting the right major fissure. (b). Sagittal image through the chest in bone windows shows innumerable sclerotic (white) lesions throughout the sternum and spine.

signs including blood pressure 158/91, pulse 76 , temperature $97.9 \mathrm{~F}\left(36.6^{\circ} \mathrm{C}\right)$. Respiratory rate was 16 per minute with $\mathrm{SpO} 297 \%$ on room air. Physical examination was essentially negative with the exception of a few respiratory rales in the lower lobes of each lung. Vaginal examination was also noncontributory. Routine labs including $\mathrm{CBC}$ and BMP were normal.

A chest radiograph revealed mediastinal and bilateral hilar adenopathy (Fig. 1). Computed tomography of the chest confirmed the mediastinal and hilar adenopathy along with scattered discrete pulmonary nodules and indistinct ground glass opacities. Additionally, there were multiple sclerotic

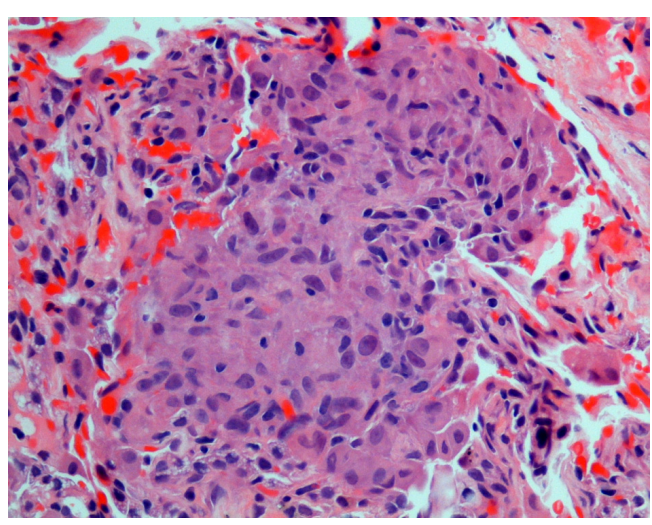

Figure 3. Histopathology from sternal biopsy (hematoxylin and eosin stain, $40 \times$ ). A high magnification view shows one of numerous intersitial noncaseating granulomas. The granulomas are well formed and composed of tightly clustered histiocytes. Similar findings were obtained from the transbronchial biopsy. lesions throughout the skeleton (Fig. 2a, b). Given her history of cancer, these findings were worrisome for metastatic disease. She underwent transbronchial biopsies and lavage as well as a sternal biopsy of a sclerotic lesion. Specimens were sent for cytology and microbiology.

The patient was confronted with the possibility of widespread metastatic disease to the chest and skeleton. She was somewhat relieved when the pathology from both biopsies revealed non-caseating granulomas consistent with sarcoidosis (Fig. 3). Special stains for AFB and fungi were negative. A serum ACE level was found to be 118 units/L (normal 7 - 46 units/L) further supporting the diagnosis. Our patient responded well to prednisone and hydroxychloroquine which were tapered/discontinued after resolution of her symptoms. She continues to undergo routine periodic evaluation by our pulmonologists in our sarcoid clinic.

\section{Discussion}

In the setting of a history of cancer, the natural first thought is metastatic disease when presented with such a case. However, one must also consider those processes that are multisystem disorders capable of mimicking more sinister pathology in the differential diagnosis.

Sarcoidosis is an idiopathic inflammatory multi-organ disease with the histological hallmark being noncaseating granulomas in affected tissues. Evidence of granulomatous inflammation in at least two organs is necessary to establish the diagnosis of sarcoidosis [1]. Both genetic and environmental causes have been implicated as potential etiologies. The most common organs affected are the lungs, eyes, and 
skin [2]. Pulmonary sarcoid can progress through multiple stages ranging from normal imaging to end stage pulmonary fibrosis. Adenopathy, pulmonary nodules, and interstitial thickening can be variably present [3]. Osseous lesions are typically lytic and rarely sclerotic and can be painful [4]. Serum ACE levels are elevated in $60 \%$ of sarcoid patients as the enzyme is produced by the granulomas [2].

First line therapy of symptomatic patients with sarcoid is glucocorticoids. However, prolonged use of steroids is subject to toxicity necessitating the use of steroid sparing agents such as anti malarials (hydroxychloroquine), minocycline or cytotoxic agents (methotrexate). Newer lines of therapy including anti-TNF agents such as infliximab are effective in improving lung function. Calcium levels should be routinely monitored in all patients with sarcoidosis as the granulomas produce 1, 25-dihydroxyvitamin D resulting in hypercalemia or hypercalciuria in about $10 \%$ [5].

A relationship between sarcoid and malignancy is controversial. Some literature discusses a sacoidosis-lymphoma syndrome in which hematologic malignancies develop within 2 years of the sarcoid diagnosis. Paraneoplastic sarcoidosis requires the development of cancer within 1 year of diagnosing sarcoid or vice versa. A relationship has also been postulated for patients with sarcoidosis and solid tumors such as melanoma and various gynecologic neoplasms including cervical and uterine [6].

Our patient presented with clinical and imaging findings pointing towards metastatic disease. Her skeletal lesions represented the rarer sclerotic presentation of sarcoid further clouding the picture. Sarcoid was diagnosed within approxi- mately one year of her cervical cancer thus raising suspicion for a possible relationship. Needless to say, she was relieved to learn that her symptoms and imaging findings were not related to widespread metastatic disease. However, the emotional distress and anxiety she suffered served as a lesson and reminder to us to always consider all possibilities.

\section{Conflict of Interest Statement}

All authors listed have no conflicts of interest.

\section{References}

1. Baughman RP, Culver DA, Judson MA. A concise review of pulmonary sarcoidosis. Am J Respir Crit Care Med. 2011;183(5):573-581.

2. Iannuzzi MC, Rybicki BA, Teirstein AS. Sarcoidosis. N Engl J Med. 2007;357(21):2153-2165.

3. Webb R, Higgins C. Thoracic Imaging. 1st ed. Philadelphia: Lippincott Willams \& Wilkins; 2005. 439-449 pp.

4. Manaster B, Roberts C, Petersilge C, et al. Diagnostic Imaging Musculoskeletal: Non-traumatic Disease. 1st ed. Canada: Amirsys; 2010. 6.40-6.43 pp.

5. Longo D, Fauci A, Kasper D, et al. Harrison's Principles of Internal Medicine. 18th ed. New York: McGraw Hill; 2012. 2805-2813 pp.

6. Cohen PR, Kurzrock R. Sarcoidosis and malignancy. Clin Dermatol. 2007;25(3):326-333. 